\title{
SELECCION DE PROCEDENCIAS DE PINO OREGON \\ (Pseudotsuga menziesii (MIRB.) FRANCO) \\ EN LA ZONA SUR DE CHILE
}

Juan José Aguirre A. (*)

Johannes Wrann H. (**)

\section{RESUMEN}

Se evalúan los resultados de tres ensayos de procedencias de Pino Oregón (Pseudotsuga menziesii (MIRB.) FRANCO) establecidos en la VIII y X Regiones del país.

A la edad de 15 años se encontraron diferencias estadísticamente significativas entre los volúmenes de las diferentes procedencias ensayadas. Los mejores resultados se obtuvieron en los dos ensayos establecidos en la zona costera de la VIII y $\mathrm{X}$ regiones. Las procedencias de la zona Noroeste de Estados Unidos, especialmente las del estado de Washington son las que presentan los mejores crecimientos.

\begin{abstract}
A Douglas fir (Pseudotsuga menziesii (Mirb.) Franco) provenance trial established in three Southern locations of Chile was assessed.

Fifteen years after the establishment, the total volumes are compared. ANOVA indicates the existence of estatistically significant differences among the tested provenances. The best results were achieved in coastal experimental sites. The north western provenances (Washington State) showed the best growth rates, among the 10 seed origins tested.
\end{abstract}

(*) Ingeniero Forestal, División Silvicultura, Instituto Forestal, Huérfanos 554 - Piso 4 - Santiago - Chile.

$\left.{ }^{(* *}\right)$ Ingeniero Forestal, División Silvicultura, Instituto Forestal, Huérfanos 554 - Piso 4 - Santiago - Chile. 


\section{INTRODUCCION}

Dentro del contexto forestal mundial, la especie Pino Oregón (Pseudotsuga menziesii (MIRB.) FRANCO) constituye una de las de mayor importancia económica. En su lugar de origen, Norteamérica, se distribuye ampliamente tanto en latitud como en longitud y ocupa una superficie de cerca de 13 millones de ha, con un volumen aproximado a 2.650 millones de metros cúbicos en pie. (USDA, 1982).

Debido a la gran capacidad de adaptarse y a las excelentes características de la madera, es una especie que se ha constituido en un recurso muy importante a nivel mundial. (UN, 1985).

En nuestro país esta especie fue introducida hace más de 90 años. Recién en 1928, se plantó en forma masiva en la zona de Traiguén. Dentro de las especies coniferas introducidas, el Pino Oregón es la segunda en importancia después de Pino Radiata. En efecto, hoy día ocupa una superficie de aproximadamente 11.000 ha, que se distribuyen preferentemente entre la IX y X Regiones (INFOR, 1987).

Previo a considerar esta especie en un programa de forestación, es de suma importancia analizar el origen de la semilla, ya que la procedencia es determinante en el éxito del establecimiento y productividad de las plantaciones.

En este trabajo se analizan la adaptabilidad y el crecimiento de 10 procedencias de Pino Oregón, establecidas por INFOR en 1968 en tres lugares de ensayo, situados en la VIII y la $\mathrm{X}$ Región.

\section{MATERIAL Y METODO}

\section{Lugares de Procedencia}

Las procedencias ensayadas provienen, en su mayoría, de las poblaciones costeras del Noroeste de los Estados Unidos y de la provincia de British Columbia, en Canadá. Sólo una corresponde a las poblaciones interiores, ya que proviene de Coconino, Arizona. En la Figura 1 se presenta el área de distribución natural del Pino Oregón excluyendo las zonas de México, (READ Y SPRACKLING, 1976) y se indican las procedencias ensayadas.

La ubicación geográfica se determinó según datos del proveedor de semillas (Silvaseed Company, Roy. Washington 98580, USA). La procedencia exacta dentro de la zona de Coconino, Arizona, se estimó de acuerdo al área de mayor ocurrencia natural del Pino Oregón.

En el Cuadro 1 se detallan los antecedentes de ubicación de los lugares de procedencia y los datos climáticos más relevantes. Estos se obtuvieron de las estaciones metereológicas más cercanas a las zonas de origen de la semilla. (U.S. DEPT. OF COMMERCE, 1959 - 1965). 
FIGURA 1

AREA DE DISTRIBUCION NATURAL DEL PINO OREGON (*)

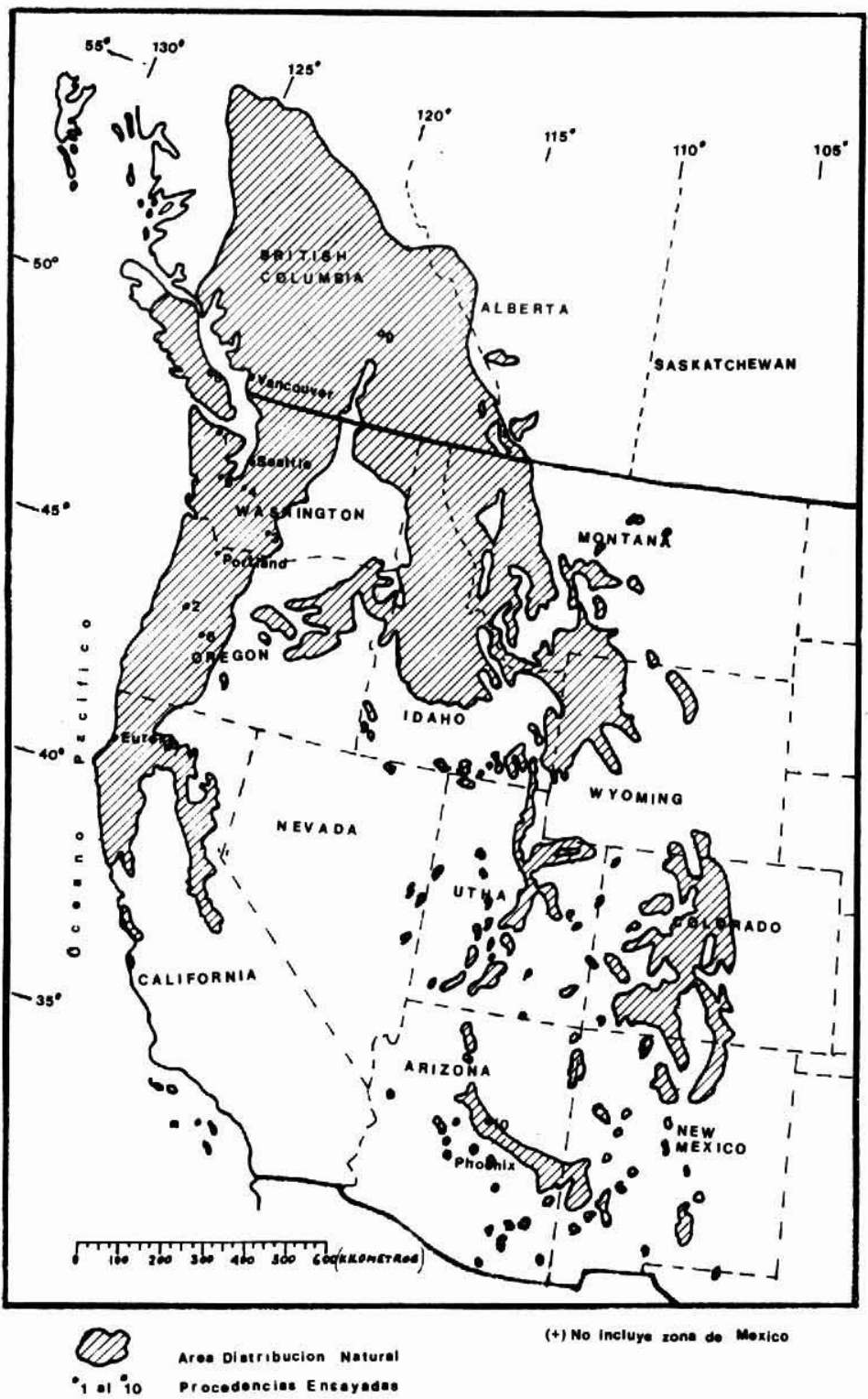

(*) Read and Sprackling, 1976 


\section{CUADRO 1 \\ UBICACION DE LOS LUGARES DE PROCEDENCIA Y ANTECEDENTES DE CLIMA}

\begin{tabular}{|c|c|c|c|c|c|c|}
\hline \multirow[b]{2}{*}{$\begin{array}{c}\text { Lugar de } \\
\text { Procedencia }\end{array}$} & \multirow[b]{2}{*}{$\begin{array}{c}\text { Código } \\
\text { Lugar de } \\
\text { Procedencia }\end{array}$} & \multirow[b]{2}{*}{ Latitud } & \multirow[b]{2}{*}{ Longitud } & \multirow[b]{2}{*}{$\begin{array}{l}\text { Altitud } \\
\text { m.s.n.m. }\end{array}$} & \multicolumn{2}{|c|}{ C 1 i m a } \\
\hline & & & & & $\begin{array}{l}\text { Precipitac. } \\
\text { mm/Año }\end{array}$ & $\begin{array}{c}\text { Temperatura } \\
\text { Media Anual } \\
{ }^{\circ} \mathrm{C}\end{array}$ \\
\hline Joyce, Washington St. & 1 & $48^{\prime} 10^{\prime} \mathrm{N}$ & $123^{\prime} 40^{\prime} \mathrm{W}$ & 23 & 1.973 & 9,6 \\
\hline Tidewater, Oregon & 2 & $44^{\prime} 20^{\prime} \mathrm{N}$ & $123^{\prime} 50^{\prime} \mathrm{W}$ & 10 & 1.480 & 10,4 \\
\hline Randle, Washington St. & 3 & $46^{\prime} 40^{\prime} \mathrm{N}$ & $121^{\prime} 50^{\prime} \mathrm{W}$ & 275 & 944 & 11,6 \\
\hline Tenino, Washington St. & 4 & $46^{\prime} 45^{\prime} \mathrm{N}$ & $122^{\prime} 40^{\prime} \mathrm{W}$ & 55 & 1.973 & 9,6 \\
\hline Pe-Ell, Washington St. & 5 & $46^{\prime} 45^{\prime} \mathrm{N}$ & $123^{\prime} 15^{\prime} \mathrm{W}$ & 55 & 1.480 & 10,4 \\
\hline Alpine Oregon & 6 & $43^{\prime} 42^{\prime} \mathrm{N}$ & $123^{\prime} 00^{\prime} \mathrm{W}$ & 200 & 944 & 11,6 \\
\hline Ashland, Oregon & 7 & $42^{\prime} 10^{\prime} \mathrm{N}$ & $122^{\prime} 45^{\prime} \mathrm{W}$ & 585 & 933 & 9,9 \\
\hline Alberni, B.C. & 8 & $49^{\prime} 24^{\prime} \mathrm{N}$ & $124^{\prime} 30^{\prime} \mathrm{W}$ & 250 & 1.048 & 9,8 \\
\hline Salmon Arms, B.C. & 9 & $51^{\prime} 00^{\prime} \mathrm{N}$ & $119^{\prime} 30^{\prime} \mathrm{W}$ & 350 & 1.369 & 9,4 \\
\hline Coconino, Arizona & 10 & $34^{\prime} 30^{\prime} \mathrm{N}$ & $111^{\prime} 25$ ' W & 1.000 & 462 & 8,9 \\
\hline
\end{tabular}

Lugares de ensayo y antecedentes de clima y suelo

En el Cuadro 2 aparecen los lugares de ensayo, su ubicación geográfica, y los correspondientes datos climáticos generales.

CUADRO 2

UBICACION Y CLIMA DE LOS LUGARES DE ENSAYO

\begin{tabular}{|l|c|c|c|c|c|c|}
\hline \multirow{2}{*}{ Lugar } & \multicolumn{3}{|c|}{ U B I C A C I O N } & \multicolumn{3}{c|}{ C L I M A } \\
\cline { 2 - 7 } & $\begin{array}{c}\text { Latitud } \\
\text { Sur }\end{array}$ & $\begin{array}{c}\text { Longitud } \\
\text { W }\end{array}$ & $\begin{array}{c}\text { Altitud } \\
\text { m.s.n.m. }\end{array}$ & Koeppen & $\begin{array}{c}\text { Temp. Media } \\
\text { Anual } \\
{ }^{\circ} \text { C }\end{array}$ & $\begin{array}{c}\text { Precipi- } \\
\text { tación } \\
\text { mm/año }\end{array}$ \\
\hline Antiquina & $38^{\circ} 06^{\prime}$ & $73^{\circ} 18^{\prime}$ & 50 & $\mathrm{C}_{\mathrm{s}} \mathrm{b}_{2}$ & 12 & 1.551 \\
Llancacura & $40^{\circ} 12^{\prime}$ & $73^{\circ} 30^{\prime}$ & 500 & $\mathrm{Cf}_{\mathrm{s}} \mathrm{b}_{2}$ & 10,6 & 1.929 \\
Trafún & $39^{\circ} 38^{\prime}$ & $79^{\circ} 51^{\prime}$ & 650 & $\mathrm{E} \mathrm{H} \mathrm{F}$ & 12 & 4.970 \\
\hline
\end{tabular}

Fuente: FUENZALIDA H., 1967. ALMEYDA E, y SAEZ F., 1958. 
En el cuadro 3 se detallan las características más relevantes de los suelos en los lugares de ensayos.

\section{CUADRO 3}

\section{SUELOS DE LOS LUGARES DE ENSAYO}

\begin{tabular}{|c|c|c|c|c|c|c|c|c|}
\hline \multirow{2}{*}{$\begin{array}{c}\text { Lugar } \\
\text { de } \\
\text { Ensayo }\end{array}$} & \multicolumn{1}{|c|}{ Origen } & Textura & $\begin{array}{c}\text { Pro- } \\
\text { fundid. } \\
\text { cm }\end{array}$ & pH & \multicolumn{2}{|c|}{$\begin{array}{c}\text { Mat. orgán. } \\
\text { \% desde Hoo. } \\
\text { Inf. a Sup. }\end{array}$} & \multicolumn{2}{|c|}{$\begin{array}{c}\text { Nutrientes (ppm) } \\
\text { Horizonte Inf. a Sup. }\end{array}$} \\
\hline Antiquina & $\begin{array}{l}\text { Ceniza Volcánica } \\
\text { y sedimentos de } \\
\text { mat. metamórfi- } \\
\text { co. }\end{array}$ & franco-arenosa & 70 & $5,7-6,0$ & $3,6-12,8$ & $5-30$ & $1-1$ & $62-186$ \\
Llancacura & $\begin{array}{l}\text { Rocas metamór- } \\
\text { ficas (Micaesquis- } \\
\text { tos). } \\
\text { Trafún }\end{array}$ & franco-arenosa & 50 & $5,3-5,6$ & $3,0-14,4$ & $5-5$ & $1-1$ & $103-180$ \\
\hline $\begin{array}{l}\text { Ceniza Volcánica. } \\
\text { franco-arenosa } \\
\text { hasta arenosa }\end{array}$ & 200 & $5,8-6,1$ & $6,2-12,4$ & $9-14$ & $1-2$ & $16-62$ \\
\hline
\end{tabular}

Fuente: MELLA, 1965; IREN, 1974; BARROS D. y ROJAS P., 1979; PERALTA M. 1975.

\section{Metodología}

\section{Disefio estadistico, establecimiento y medición de los ensayos}

El diseño experimental empleado fue el de bloques al azar con tres repeticiones. La unidad experimental corresponde a una parcela de 49 plantas, con un espaciamiento de $3 \times 3 \mathrm{~m}$; de los cuales sólo se miden las 25 centrales.

La plantación se realizó en Julio de 1968, con plantas a raiz desnuda, con dos temporadas en el vivero sin repique $(2: 0)$, con un tamaño medio de $18 \mathrm{~cm}$ y una relación tallo: raiz de 1.1:1.

Las parcelas se midieron a los 2, 5, 10 y 15 años, excepto en Trafún, en donde no se realizó la tercera medición. En este informe se analizan los datos obtenidos en el último control, en el cual se midieron los siguientes parámetros: Supervivencia, Altura Total y DAP.

\section{Metodología de análisis}

Con los datos obtenidos en cada ensayo, se calcularon los parámetros medios de: altura total en $\mathrm{m}$, DAP en $\mathrm{cm}$, número de árboles por hectárea y área basal por hectárea $\left(\mathrm{m}^{2} / \mathrm{ha}\right)$. En base a los parámetros de cada árbol vivo se calculó el volumen cúbico con corteza, según la fórmula de OPIE (1976). La función de volumen es la siguiente:

$$
\log _{10}\left(D^{2} H / V\right)=4,762-5613 /(D+127)^{2}
$$


Se realizaron análisis de varianza para las variables de estado: altura promedio y volumen por hectárea. Debido a que en los ensayos de Antiquina y Trafún se habian destruido algunas parcelas, previo a realizar el análisis de varianza se utilizó la metodología que describe OSTLE (1964) y STEEL AND TORRIE (1960) para estimar los datos faltantes.

Al aplicar la Prueba de Bartlett se determinó que existía heterogeneidad en las varianzas, por lo que fue necesario realizar una transformación logarítmica (base 10), de acuerdo a lo que recomienda SACHS (1978).

Con el fin de determinar la existencia de interacciones entre los lugares de ensayo y las procedencias, se aplicó un análisis de varianza para series de experimentos, según lo descrito por PANSE y SUKATHME (1963) y COCHRANE y COX (1965).

\section{RESULTADOS}

\section{Parámetros Medios y Volumen}

Los resultados de los parámetros altura y DAP promedio, número de árboles por hectárea, área basal y volumen por hectárea se entregan en los Cuadros 4,5 y 6 para los lugares de ensayo Antiquina, Llancacura y Trafún respectivamente. Las procedencias están ordenadas de mayor a menor volumen, en cada lugar de ensayo.

CUADRO 4

PROCEDENCIAS DE PINO OREGON CRECIMIENTO A LA EDAD DE 15 AÑOS Lugar de Ensayo: Antiquina

\begin{tabular}{|l|c|c|c|c|c|}
\hline \multicolumn{1}{|c|}{ Procedencia } & $\begin{array}{c}\text { Altura } \\
\text { Promedio } \\
(\mathbf{m})\end{array}$ & $\begin{array}{c}\text { DAP } \\
\text { Promedio } \\
\mathbf{( c m )}\end{array}$ & $\begin{array}{c}\text { Número } \\
\mathbf{A r b o l e s} \\
\mathbf{( / h a )}\end{array}$ & $\begin{array}{c}\text { Area } \\
\mathbf{B a s a l} \\
\left(\mathbf{m}^{2} / \mathbf{h a}\right)\end{array}$ & $\begin{array}{c}\text { Volumen } \\
\left(\mathbf{m}^{\mathbf{3}} / \mathbf{h a} \mathbf{)}\right.\end{array}$ \\
\hline PE ELL (Wash. St.) & 9,9 & 20,3 & 1044 & 34,7 & $147,5 \mathrm{a}$ \\
RANDLE (Wash. St.) & 8,9 & 19,9 & 1022 & 32,9 & $127,7 \mathrm{a}$ \\
TIDEWATER (Oregon) & 9,1 & 19,4 & 963 & 31,1 & $119,3 \mathrm{a}$ \\
JOYCE (Wash. St.) & 9,1 & 20,3 & 778 & 27,8 & $118,5 \mathrm{a}$ \\
ALPINE (Oregon) & 8,6 & 20,0 & 1022 & 33,5 & $117,3 \mathrm{a}$ \\
ASHLAND (Oregon) & 9,0 & 18,7 & 830 & 24,4 & $93,6 \mathrm{a}$ \\
ALBERNI (B.C.) & 7,3 & 15,3 & 733 & 18,2 & $70,2 \mathrm{~b}$ \\
TENINO (Wash. St.) & 7,3 & 15,1 & 711 & 16,7 & $58,6 \mathrm{~b}$ \\
SALMON ARMS(B.C.) & 6,6 & 15,5 & 726 & 16,6 & $54,0 \mathrm{~b}$ \\
COCONINO (Arizona) & 6,3 & 14,3 & 519 & 11,3 & $34,9 \mathrm{~b}$ \\
\hline
\end{tabular}

Las procedencias que no presentan diferencias significativas entre si se señalan con una misma letra. (Test de Duncan, $95 \%$ de confianza). 


\section{CUADRO 5 \\ PROCEDENCIAS DE PINO OREGON \\ CRECIMIENTO A LA EDAD DE 15 AÑOS \\ Lugar de Ensayo: Llancacura}

\begin{tabular}{|l|c|c|c|c|c|}
\hline \multicolumn{1}{|c|}{ Procedencia } & $\begin{array}{c}\text { Altura } \\
\text { Promedio } \\
(\mathbf{m})\end{array}$ & $\begin{array}{c}\text { DAP } \\
\text { Promedio } \\
\mathbf{( c m )}\end{array}$ & $\begin{array}{c}\text { Número } \\
\text { Arboles } \\
\text { (/ha) }\end{array}$ & $\begin{array}{c}\text { Area } \\
\text { Basal } \\
\left(\mathbf{m}^{2} / \mathbf{h a}\right)\end{array}$ & $\begin{array}{c}\text { Volumen } \\
\left(\mathbf{m}^{\mathbf{3}} / \mathbf{h a}\right)\end{array}$ \\
\hline JOYCE (Wash. St.) & 11,5 & 23,5 & 800 & 36,1 & $164,7 \mathrm{a}$ \\
COCONINO (Arizona) & 11,1 & 21,6 & 844 & 32,6 & $146,2 \mathrm{a}$ \\
TIDEWATER (Oregon) & 10,6 & 22,2 & 682 & 26,1 & $112,3 \mathrm{a}$ \\
TENINO (Wash. St.) & 10,3 & 20,7 & 667 & 24,0 & $103,1 \mathrm{a}$ \\
RANDLE (Wash. St.) & 10,2 & 23,2 & 548 & 21,8 & $97,4 \mathrm{a}$ \\
ASHLAND (Oregon) & 9,9 & 18,8 & 741 & 22,4 & $94,1 \mathrm{ab}$ \\
ALPINE (Oregon) & 9,6 & 19,4 & 637 & 22,2 & $93,3 \mathrm{~b}$ \\
ALBERNI (B.C.) & 8,4 & 18,2 & 385 & 12,3 & $52,0 \mathrm{~b}$ \\
PE ELL (Wash. St.) & 9,1 & 18,7 & 859 & 23,6 & $50,0 \mathrm{~b}$ \\
SALMON ARMS (B.C.) & 5,1 & 11,0 & 563 & 5,9 & $14,1 \mathrm{c}$ \\
\hline
\end{tabular}

CUADRO 6

PROCEDENCIAS DE PINO OREGON

CRECIMIENTO A LA EDAD DE 15 AÑOS

Lugar de Ensayo: Trafún

\begin{tabular}{|c|c|c|c|c|c|}
\hline Procedencia & $\begin{array}{c}\text { Altura } \\
\text { Promedio } \\
\text { (m) }\end{array}$ & $\begin{array}{c}\text { DAP } \\
\text { Promedio } \\
\text { (cm) }\end{array}$ & $\begin{array}{c}\text { Número } \\
\text { Arboles } \\
\text { (/ha) }\end{array}$ & $\begin{array}{c}\text { Area } \\
\text { Basal } \\
\left(\mathrm{m}^{2} / \mathrm{ha}\right)\end{array}$ & $\begin{array}{c}\text { Volumen } \\
\left(\mathrm{m}^{3} / \mathrm{ha}\right)\end{array}$ \\
\hline RANDLE (Wash. St.) & 8,5 & 14,2 & 667 & 13,5 & 54,2 a \\
\hline PE ELL (Wash. St. & 8,5 & 13,5 & 696 & 12,4 & 49,6 a \\
\hline SALMON ARMS (B.C.) & 9,3 & 13,6 & 733 & 12,7 & 49,6 a \\
\hline JOYCE (Wash. St.) & 9,3 & 13,3 & 682 & 11,0 & 49,3 a \\
\hline COCONINO (Arizona) & 9,1 & 15,2 & 519 & 11,3 & 48,4 a \\
\hline TIDEWATER (Oregon) & 8,2 & 11,6 & 978 & 11,8 & 41,8 a \\
\hline ASHLAND (Oregon) & 7,4 & 12,7 & 770 & 11,0 & $38,4 \mathrm{~b}$ \\
\hline TENINO (Wash. St.) & 7,7 & 11,2 & 867 & 9,6 & $31,0 \mathrm{~b}$ \\
\hline ALPINE (Oregon) & 7,4 & 11,8 & 682 & 8,3 & $29,2 \mathrm{~b}$ \\
\hline ALBERNI (B.C.) & 7,4 & 11,9 & 578 & 7,1 & $25,6 \mathrm{~b}$ \\
\hline
\end{tabular}


Con el fin de comparar más fácilmente el comportamiento de las procedencias con cada uno de los lugares de ensayo, los resultados de altura y volumen también se presentan en forma gráfica (Figuras 2 y 3 ).

FIGURA 2

\section{ALTURAS MEDIAS POR PROCEDENCIA Y LUGAR DE ENSAYO \\ Especie: Pino Oregon \\ Edad: 15 años}

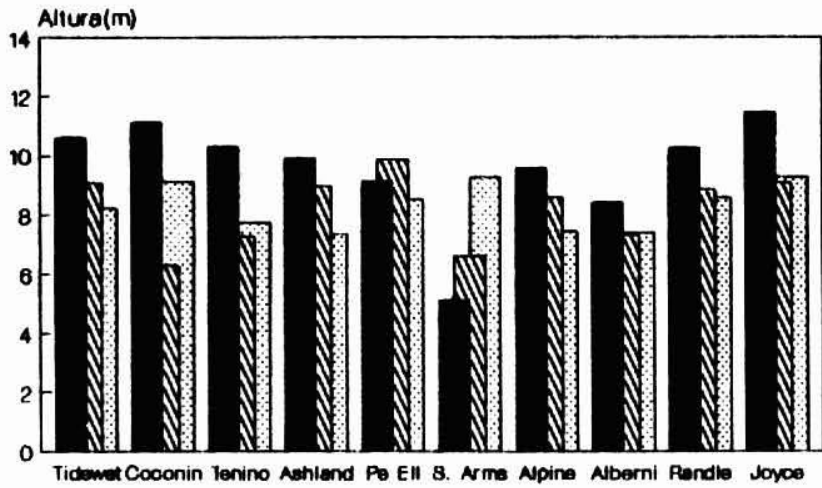

LUGAR DE ENSAYO

Llenceoura AIIV Antiquine

FIGURA 3

VOLUMEN POR PROCEDENCIA Y

LUGAR DE ENSAYO

Especie: Pino Oregón

Edad: 15 años

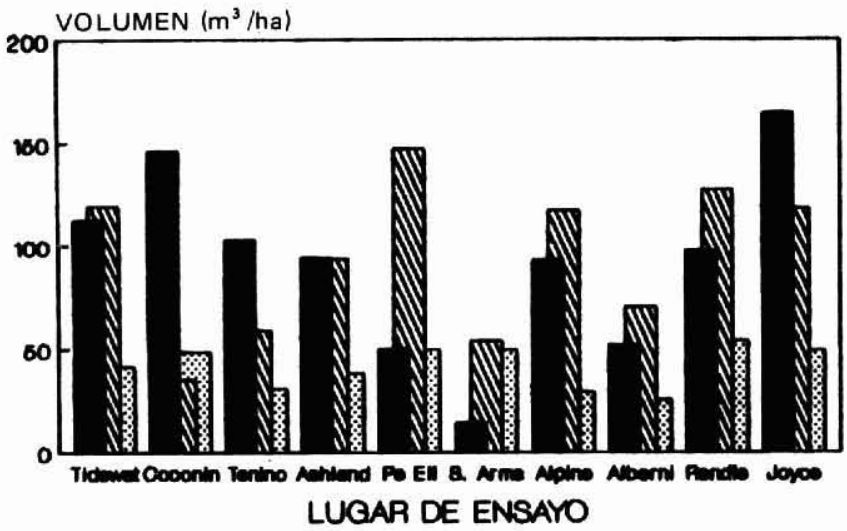

Llenosecure IIIII Antlowine Tratun 


\section{Análisis Interacción Sitio - Procedencia}

Al aplicar el análisis para series de experimentos entre los tres lugares de ensayo (PANSE V. G. y SUKATHME P.V., 1963; COCHRANE y COK, 1965) se determinó que existe interacción entre éstos y las procedencias (nivel de confianza 99\%) para los parámetros altura media, volúmenes y sobrevivencia (Cuadro 7). Este análisis no arrojó diferencias significativas entre procedencias para todos los parámetros analizados, pero sí entre los lugares de ensayo. Sin embargo, según se indica en los Cuadros anteriores ( $\left.\mathrm{N}^{4}-5-6\right)$, al analizar puntualmente cada lugar de ensayo, se determinaron diferencias significativas entre procedencias para el parámetro volumen $(95 \%$ de confianza)

\section{CUADRO 7 \\ RESULTADO PARA SERIES DE EXPERIMENTOS \\ Especie: Pino Oregon}

\begin{tabular}{|l|c|c|c|c|c|}
\hline Fuente Var. & GL & Altura media & Area Basal & Volumen & Sobrevivenc. \\
\hline Lugar & 2 & $*$ & $* *$ & $* *$ & $* *$ \\
Procedencia & 9 & N S & N S & N S & N S \\
Interacción & 18 & $* *$ & N S & $* *$ & $* *$ \\
\hline
\end{tabular}

* Significativo al nivel $95 \%$ de confianza.

** Significativo al nivel $99 \%$ de confianza.

\section{DISCUSION}

El clima como factor de adaptación

Para comparar el clima de los lugares de procedencia y de los ensayos Llancacura y Antiquina, se superpusieron los climatogramas poligonales o hiterógrafos (CALDERON S., 1966) de cada lugar de procedencia con los del ensayo.

En general se observa una mejor adaptación de la especie cuando las condiciones climáticas de los lugares de procedencia y del ensayo son similares. Sin embargo en Llancacura esta tendencia se observa con menor claridad. Por otra parte llama la atención que en dicho lugar la procedencia de Coconino, cuyas condiciones de clima son muy distintas a las del ensayo, presente resultados satisfactorios.

Al comparar los resultados de los ensayos establecidos en Antiquina y Llancacura, se observa que los mejores corresponden a la procedencia Joyce (Washington) que presenta condiciones climáticas semejantes a los de los lugares de ensayo. (Figura 4). 


\section{FIGURA 4 \\ CLIMATOGRAMAS : \\ JOYCE, ANTIQUINA Y LLANCACURA}

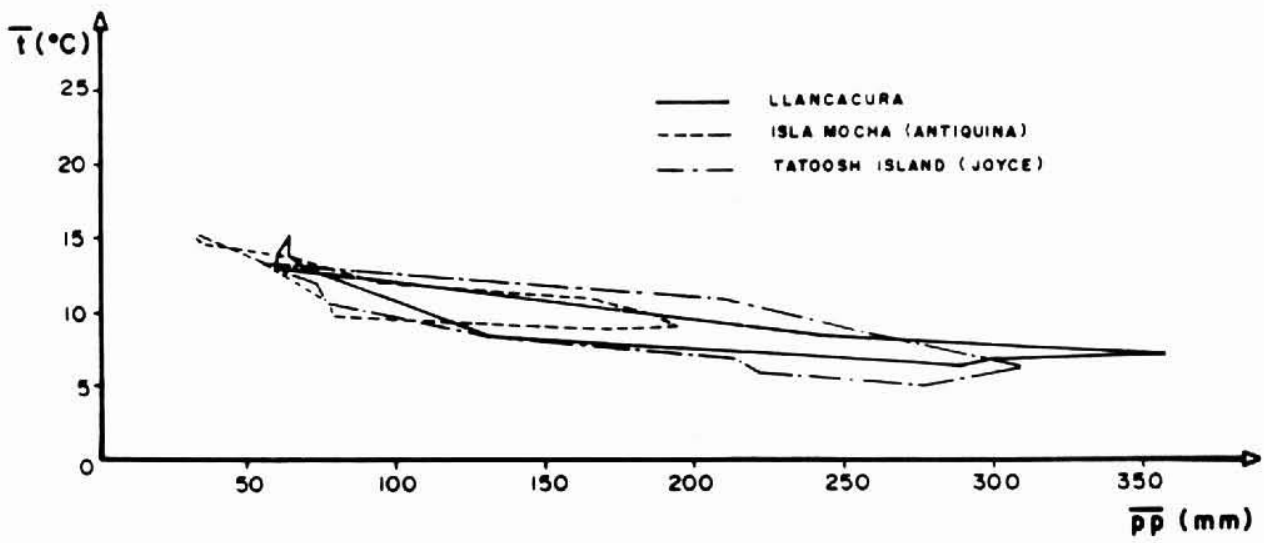

\section{Resultados en Antiquina (Arauco)}

Los crecimientos medios en volumen de las 10 procedencias de Pino Oregón ensayadas en Antiquina difieren entre sí significativamente. Las 6 procedencias típicamente costeras, identificadas con los números $1,2,4$ y 5 , que provienen de una zona climática similar al lugar de ensayo, presentan en general una buena adaptación, a pesar de haber crecido sometidas a una fuerte competencia hasta los 10 años de edad, ya que la plantación fue invadida por zarzamora (Rubus sp.). En el crecimiento inicial en altura se observaron diferencias significativas hasta la edad de 10 años (WRANN, 1979).

El suelo en el lugar de ensayo es fértil, profundo y bien drenado, lo cual favorece el desarrollo de la especie. CONTRERAS (1982) en un estudio realizado en la provincia de Valdivia. determinó que la profundidad del horizonte A y el drenaje son las variables del suelo más importantes para determinar el índice de sitio de Pino Oregón.

A pesar de esta buena adaptación, los volúmenes estimados para las procedencias de mejor crecimiento son inferiores a los estimados en plantaciones comerciales en la zona de Villarrica (CONTRERAS y SMITH, 1973). Es necesario mencionar que se han empleado distintas funciones de volumen, en cada caso. Según dicho estudio, a los 15 años el volumen era de $188 \mathrm{~m}^{3} / \mathrm{ha}$.

Otro estudio de procedencias de Pino Oregón en la zona de Valdivia determinó volúmenes entre 343 a $471 \mathrm{~m}^{3} /$ ha a la edad de 17 años (DROPPELMAN, 1986), reflejando crecimientos muy superiores a los obtenidos en este estudio.

\section{Resultados en Llancacura}

Llancacura presenta los mejores resultados de adaptación y crecimiento, aunque sólo levemente superiores a los del ensayo de Antiquina.

En este ensayo también se observan diferencias significativas entre procedencias, al comparar su crecimiento en volumen. Al igual que en Antiquina, las procedencias con mejor desarrollo son Joyce, Pe-Ell y Tidewater, todas de la zona del NW de Estados Unidos. 
La procedencia de Coconino, Arizona, también presenta buenos resultados, a pesar de que las condiciones climáticas en su lugar de origen son muy diferentes. Es probable que este hecho sea algo excepcional, ya que normalmente las procedencias interiores son de crecimiento más lento (tal como aparece demostrado en el ensayo de Antiquina) aunque más resistentes a bajas temperaturas y sequía.

Siguiendo la misma tendencia del ensayo anterior, las diferencias en altura entre procedencias tienden a igualarse a medida que han pasado los años. Los mejores resultados también son inferiores a los obtenidos por DROPPELMAN (1986) en Valdivia, pero semejantes a los logrados en Villarrica en el estudio de CONTRERAS y SMITH (1973).

\section{Resultados en Trafún}

En este lugar, las procedencias ensayadas presentan un crecimiento en volumen bastante inferior, en relación a los otros dos ensayos. Esto se puede explicar por las condiciones en que crecieron inicialmente las plantas, las que estuvieron sometidas a una fuerte competencia del rebrote de la vegetación nativa, lo cual restringe el crecimiento y afecta las tasas de supervivencia. Además, el sitio del ensayo está a mayor altitud y por lo tanto presenta una temperatura media anual inferior y está expuesto a heladas más severas y prolongadas.

\section{CONCLUSIONES}

De los ensayos analizados se concluye que las procedencias costeras del Noroeste de los Estados Unidos son las que mejor se adaptan en la zona centro sur de Chile, ya sea en condiciones costeras o de precordillera. Estos resultados, especialmente los obtenidos en Antiquina, confirman que la similitud climática es un buen punto de partida para la selección de procedencias. En efecto, numerosas investigaciones se han realizado en relación a este tema y la mayoría de ellas señala que hay diferencias muy significativas de desarrollo entre procedencias de diferentes regiones.

Comparando los ensayos analizados, la mejor área para el crecimiento de la especie resulta ser la Cordillera de la Costa de la X Región. Sin embargo, estos resultados son superados por los que obtuvo DROPPELMANN (1986) en Valdivia, pero estas diferencias pueden deberse a las técnicas de establecimiento empleadas.

Especificamente, las mejores procedencias corresponden a Joyce (Washington), Pe-Ell (Washington) y Randle (Washington), además de la de Coconino (Arizona), que presenta buenos resultados en Llancacura.

Cualquier acción encaminada a utilizar esta especie como alternativa de forestación debe considerar necesariamente el origen de la semilla, pues los mejores resultados de crecimiento y supervivencia se han obtenido con aquellas que provienen de lugares similares a los de las zonas ensayadas.

\section{RECONOCIMIENTOS}

Este trabajo ha sido preparado con los antecedentes del Proyecto Introducción de Especies Forestales, financiado por la Corporación de Fomento de la Producción (CORFO).

Los autores agradecen al señor Juan Carlos Bañados, por su participación en el análisis estadistico de los resultados. 


\section{REFERENCIAS BIBLIOGRAFICAS}

1. ALMEYDA A., ELIAS y SAEZ S., FDO. Recopilación de datos climáticos de Chile y Mapas sinópticos respectivos. Ministerio de Agricultura. Santiago Chile, 1958.

2. BARROS, R., DANIEL y ROJAS, V. PATRICIO, 1979. Evaluación de Parcelas Experimentales de Introducción de Especies. Tesis. Facultad de Ciencias Forestales, Universidad de Chile.

3. CALDERON S. SERGIO, 1966: Estudio preliminar fitoclimático de supervivencia en la introducción de algunas especies exóticas. Tesis, Facultad de Ciencias Forestales, Universidad de Chile, Santiago.

4. COCHRANE, W.G. y COX, G.M. 1965. Diseños Esperimentales Ed. F. Trillas México 661 p.

5. CONTRERAS A. J.M. y SMITH, G. BYRON. 1973. Estudio preliminar de Incremento y Rendimiento de Pino Oregón en la Región Sur de Chile. Tesis. Facultad de Ingeniería Forestal, Universidad Austral de Chile, Valdivia.

6. CONTRERAS C. CARLOS. 1982. Indices de sitio para Pino Oregón en la provincia de Valdivia y sus relaciones con los sitios para Pino Insigne. Tesis, Facultad de Ingeniería Forestal. U. Austral de Chile. Valdivia.

7. FUENZALIDA H. 1967. Clima. En: Geografía Económica de Chile. CORFO. Edit. Universitaria, 1967 (p. 98 - 152).

8. DROPPElmanN, FElmer, J. 1986. Evaluación de un ensayo de Procedencias de Pino Oregón (Pseu. dotsuga menziesii (MIRB) Franco) de 17 años de edad (Fundo Las Palmas, Valdivia). Tesis. Facultad de Ciencias Forestales, Universidad Austral de Chile. Valdivia.

9. IREN. 1974. Estudio Integrado de los Recursos Naturales Renovables. Provincia de Valdivia. Corporación de Fomento de la Producción. 195 p.

10. INFOR. 1986. Especies Forestales Exóticas de Interés Económico para Chile. Corporación de Fomento de la Producción, Gerencia de Desarrollo AF 86/32.

11. INFOR. 1987. Bolet ín Estadístico $\mathrm{N}^{\circ}$ 6. Santiago. Chile.

12. MELLA, A. ARNOLDO. 1965. Estudio Agrológico de los Predios Antiquina, Lluncao y Paicaví. Departamento de Agrología. Servicio Agrícola Ganadero. Santiago.

13. PANSE V. G. y SUKHATME P.V., 1963. Métodos estad ísticos para investigadores agrícolas. Ed. Fondo de Cultura Económica. México. 350 p.

14. OPIE, J.E. 1976. Volume Functions for Trees of all Sizes. In: Forestry Technical Papers $\mathrm{N}^{\circ} 25$. Forest Commission. Victoria, Australia (p. $27-30$ ).

15. OSTLE, BERNARD. 1964. Estadística Aplicada. Edit. Limusa. Wiley S.A. México.

16. PERALTA, P. MARIO. 1975. Ecologia y Silvicultura del Bosque Nativo Chileno. Boletín Técnico $\mathrm{N}^{\circ} 31$. Fac. Cs. Forestales, Universidad de Chile, Santiago.

17. READ RALPH A., SPRACKLING, JOHN A. 1976. Douglas fir in Eastern Nebraska: A Provenance Study USDA, For Serv. Res. Pap. R.M. 178. 10 p. Rocky Mountain For. Exp. Sta. Fort Collins C.

18. ROCUANT, L.R. 1970. Importancia del origen de la Semilla. Chillán, U. de Concepción. Nota Informativa $N^{\circ} 8$.

19. STEEL, G. D.R. and TORRIE, J. 1960. Principles and Procedures of Statistics with Special reference to the Biological Sciences. Mc. Graw-Hill N.Y. 481 p.

20. SACHS L. 1978. Angewandte Statistik. SPRINGER. V., N. York. 552 p.

21. U.S. DEPT. OF COMMERCE 1959: World weather Records 1941-50 U.S. Government Printing Office, Washington D.C.

22. U.S. DEPT. OF. COMMERCE 1965: World weather Records 1951-60. U.S. Government Printing Office, Washington D.C.

23. U.S.D.A. FOREST SERV. 1982. On analysis of the Timber Situation in the U.S. 1952 - 2030. For. Res. Rep. 23. Wash. D.C. 499 p.

24. 1985. The Forest Resources of the ECE Region (Europe, USSR. NA) United Nations, New York. $233 \mathrm{p}$.

25. WRANN, H., J. 1979. Resultados de Adaptación de 10 Procedencias de Pino Oregón (Pseudotsuga menziesii (Mirb.) Franco) en Antiquina (Arauco) y Llancacura (Osorno). Instituto Forestal. Informe Interno no publicado. 